\title{
West Syndrome Case of Unknown Cause of Difficult Treatment
}

\author{
Case Report
}

Berríos C*

Pediatrician, San Juan De Dios Esteli Hospital, Nicaragua.

Abstract

West syndrome is an age-dependent epileptic encephalopathy, is a kind of catastrophic epilepsy in the first year of life, characterized by the electroclinical triad of epileptic spasms, delayed psychomotor development, and hypsarrhythmia on the electroencephalogram, although one of these elements may be absent [1].

Its pathophysiology remains unknown and presents a wide range of etiologies, under which can be classified into: genetic, structural/metabolic and unknown cause (cryptogenic). The prognosis depends on: (a) the cause, which gives origin to the attacks (the complex malformation forms being more severe); (b) the EEG pattern(s); (c) the appearance of seizures prior to the spasms; and (d) the rapid response to treatment. Currently, the first-line treatment includes the adrenocorticotropic hormone ACTH and vigabatrin. In the near future the gold standard could be the development of new therapies that target specific pathways of pathogenesis [2]. Due to its high mortality rate and refractoriness to antiepileptic drugs Conventional, is considered a type of "catastrophic epilepsy".

Keywords: West Syndrome; Hypsarrhytmia; Infantile Spams.

\section{Introduction}

In 1841 Dr. William James West (1793-1848), an English physician, first described in an article published by the Lancet, a clinical pattern of new convulsions present in his 4-monthold son, characterized by starting with a subtle head drop and progressing to clusters of myoclonic spasms occurring many times daily, accompanied by the regression of development of his previously normal child [3].

The term West Syndrome (WS) refers to a form (a subset) of Infantile Spasms Syndrome (ISs), characterised by the combination of clustered spasms and hypsarrhythmia on an EEG and delayed brain development or regression [currently, it is no longer required that delayed development occur before the onset of spasms]. Less usually, spasms may occur singly rather than in clusters [infantile spasms single-spasm variant (ISSV)] [2].

Infant spasms and West Syndrome represent the most common type of epilepsy in the first year of life. The incidence varies from 2 to 5/10,000 live births, with onset in the first year of life in
$90 \%$ of those affected. This translates into approximately 2,000 to 2,500 new cases per year in the United States. In Europe, the prevalence at birth estimated to date is $3.7 / 100,000$ children.

The peak age of onset is between 3 and 7 months; onset after 18 months is rare, though onset up to 4 years of age has been reported [4].

\section{Classification}

West syndrome is classified as symptomatic and cryptogenic. In the first case, there is an identified etiology and/or a significant delay in development at the time of onset of spasms; while in the second it is not possible to identify a precipitating etiology. An idiopathic group was then added to include those patients with no known cause and no symptoms or neurological abnormalities. This classification was revised in 2010 by the ILAE Committee on Classification and Terminology, replacing the terms "idiopathic", "symptomatic" and "cryptogenic", by the terms "genetic", "structural / metabolic" and "unknown cause" respectively [5].

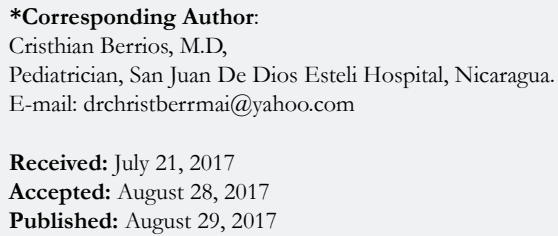

Copyright: Berríos $\mathbf{C}^{\circ}$ 2017. This is an open-access article distributed under the terms of the Creative Commons Attribution License, which permits unrestricted use, distribution and reproduction in any medium, provided the original author and source are credited. 


\section{Etiology}

According to the literature the West Syndrome etiology is very varied identifying the cause in more than $50 \%$ of the cases. According to the United Kingdom Infantile Spams Study conducted in 2010, the most common causes of infantile spasms in order of frequency were hypoxic-ischemic encephalopathy $(10 \%)$, chromosomal abnormalities $(8 \%)$, malformations $(8 \%)$, stroke Perinatal (8\%), tuberous sclerosis complex $(7 \%)$ and periventricular leukomalacia or cerebral haemorrhage (5\%).

Other etiologies include cortical dysplasia, meningoencephalitis, trauma, other neurocutaneous disorders such as linear sebaceous nevus, pigmentary incontinence, Ito syndrome and type I neuroibromatosis, congenital infections (TORCH) and inborn errors of metabolism. In children, West syndrome may also be caused by mutations of the ARX gene, often associated with ambiguous genitalia. As a result of increased use of magnetic resonance imaging and PET (Positron Emission Tomography), fewer spasms (approximately 10-15\%) are labeled as cryptogenic, and more are attributed to an underlying brain anomaly not previously detected [5].

\section{Pathogenesis}

Despite the time that has elapsed since its recognition, the biological basis of the West syndrome is not yet fully determined. It is postulated that they are the result of a non-specific injury at a critical point in the ontogenetic development of the brain, where several etiopathogenic factors, whether structural, metabolic, genetic, inflammatory or even neuroimmune, seem to be involved.

There is evidence that the underlying mechanism is due to alterations in the key genetic regulatory pathways of brain development, specifically the regulatory gene network for dorsalventral development of the anterior GABAergic brain and abnormalities in the gene expressed at the synapse. Children with these genetic associations also have phenotypes beyond epilepsy, including dysmorphic features, autism, movement disorders, and systemic malformations. However, the therapeutic response to ACTH and high doses of steroids, as well as occasional clinical reports of cytokine abnormalities or infectious and autoimmune etiologies, have raised the possibility that neuroinflammatory pathways may be involved in at least a number of patients.

The mTOR pathway appears to play a central role in the convergence of several signaling mechanisms and may represent a promising avenue for future therapeutic interventions [5].

\section{Clinical Manifestations}

West syndrome is characterized by the electroclinic triad of Epileptic spasms, retardation of psychomotor development and electroencephalographic pattern of hypsarrhythmia.

Epileptic spasm or clinical spasm consists of a brief abrupt contraction of less than 2 seconds duration, followed by a sustained tonic contraction of less intensity, usually 2 to 10 seconds duration, involving the muscular groups of the neck, trunk and Extremities, usually symmetrically and synchronously, although there may be several clinical patterns. Less frequently the contraction phase lasts less than 0.5 seconds and occurs without the tonic phase [5].

The spasms may be of the lexor, extensor or mixed type, without suggesting etiology or affecting the prognosis, with more than one being present in the same patient. However, when they are asymmetrical $(5-25 \%)$ they guide a structural abnormality of the brain [5].

About $80 \%$ of the spasms occur in groups or "clusters" of several minutes duration, where they can present from 2 to 125 in an episode, with an average of 13 per minute. They usually occur before awakening or before sleep, with a similar frequency between day and night, although they rarely occur during sleep [5].

In general there is no obvious precipitating circumstance or stimulus, although some factors that are posed as crisis inducers are the difficulty in sleeping and nocturnal awakenings during the manipulation of the child, loud noises, feeding, infections, fever, Emotion, hunger and extreme environmental temperatures [5].

During the seizure, several clinical phenomena can be observed, such as behavioral changes, gaze deviation, nystagmus or changes in the respiratory pattern. Often the child may cry or cry after the ictal phase, although they are not considered part of the seizure. After the crisis there may be irritability or transient hyporesponsiveness. In addition, $30-45 \%$ of patients may present with other seizures, such as partial, myoclonic, tonic and tonicclonic seizures [5].

Delay or Regression in Neurodevelopment is usually severe, with evident motor and cognitive manifestations in most patients, including mental and speech retardation, autistic traits and somotordysraxia. Psychomotor retardation may precede the onset of spasms, but may also occur simultaneously or be late [5].

\section{Electroencephalographic Characteristics}

The characteristic feature in west syndrome is hypsarrhythmia, a disorganized interictal pattern consisting of slow waves and high voltage, random points in all cortical areas. This pattern is observed while the child is awake and in non-REM sleep, whereas during REM sleep there is a marked decrease and may even disappear. In addition to the classical pattern already described, variants of hypsarrhythmia have been grouped with the term "modified hypsarrhythmia" or "atypical", which is characterized by greater hemispheric synchronization and symmetry and is observed in about two-thirds of the patients. These variants include hypsarrhythmia with increased interhemispheric synchronization, asymmetric or unilateral pattern, abnormal discharges by focal abnormalities, attenuation episodes of activity, and the pattern of high voltage slow waves without spikes [5].

The most common ictal pattern consists of a three-phase, positive and large-amplitude slow wave that correlates with the current spasm, followed by a flattening by a generalized abrupt attenuation of the activity. There is no correlation between the ictal pattern and the type of spasm. It should be noted that focal or lateralized EEG findings may indicate the presence of a structural brain injury [5]. 


\section{Differential Diagnosis}

Differential diagnoses of SW are those pathologies that may be confused with clinical spasms, such as gastroesophageal relapse, colic, excessive jitters, exaggerated Moro's reminiscences, benign neonatal sleep myoclonus, tonic seizures of early childhood, and benign myoclonic epilepsies And serious. It should be borne in mind that these conditions may also occur in patients who have previously had or currently have West Syndrome.

\section{Diagnostic Evaluation}

The initial evaluation begins with a detailed medical history and physical examination. The diagnosis is made with a compatible clinical picture confirmed by EEG.

The recommended method is to make a video EEG monitoring 24 hours, in order to capture an ictal event and include a sleepwake cycle complete, although in some cases it may be suficient a study of two or four hours. In case the initial evaluation is not diagnostic, the monitoring can be extended or repeated within 1-2 weeks [5].

Established the diagnosis the next step is to identify the etiology. The study with neuroimaging is essential, since it allows to confirm the etiology in approximately $70 \%$ of the cases, being the initial technique of choice the magnetic resonance (MRI), since it allows detecting cerebral malformations, cerebral atrophy, delay in myelination and other focal lesions not visible on computed tomography (CT). It can also provide information on the prognosis, especially in relation to motor development; the patients with MRI without alterations have better results when compared with those with lesions [5].

In cases where clinical examination or neuroimaging makes it possible to suspect a genetic disorder, it is recommended to perform genetic tests, for example in cases of Down syndrome or lysencephaly.

If it is not possible to identify an etiology after clinical evaluation, EEG and MRI, or in cases of early-onset spasms, association with other types of seizures and severe mental retardation at onset, or poor outcome after spasm control, It is recommended to perform a metabolic screening; Levels of transaminases, lactate, pyruvate, ammonia, organic acids in urine and serum amino acids, as well as analysis of the cerebrospinal fluid to measure glucose, protein, amino acids, lactate and pyruvate levels. The combination of metabolic study results (serum and cerebrospinal fluid) with genetic tests can determine the etiology in an additional 10\% of the cases [5].

Another recommended study is PET, useful in the identification of anomalies not detected in MRI or CT, and considered in medically intractable cases when the presence of focal abnormalities in the EEG or findings at the clinical examination suggest possible epileptogenic zones that could be susceptible to resection Surgical [5].

\section{Treatment}

West syndrome treatment continues to be empirical because of the difficult understanding of its pathophysiology and resistance to anticonvulsant drugs.

\section{First-line Therapies}

In general the treatment is divided into two large groups; Children with tuberous sclerosis (ET), where the drug is preferably vigabatrin, and carriers of all other etiologies, where the therapy of choice is the hormone corticotropin (ACTH).

Recent studies suggest that early initiation of treatment, either with hormone therapy or vigabatrin, improves long-term cognitive outcomes. In addition, it has been observed that ACTH is superior to vigabatrin in terms of cognitive outcome in cryptogenic groups.

\section{Second-line Therapies}

Different corticosteroids and AEDs can be used as second-line alternatives when first-line drugs are ineffective or contraindicated.

Corticosteroids: hydrocortisone, prednisone, prednisolone and dexamethasone.

AEDs: The evidence for other AEDs is very limited compared to vigabatrin. Topiramate, valproic acid, sultiamo, zonisamide, pyridoxine, nitrazepam, levetiracetam, lamotrigine and ketogenic diet.

\section{Surgical Treatment}

About $25-40 \%$ of patients continue to have spasms despite intensive pharmacotherapy, and express psychomotor retardation, so that they may be candidates for surgery. Focal cortical surgical resections have resulted in cessation of spasms and developmental normalization. Successful functional hemispherectomies have been performed in cases of severe hemispheric anomalies such as Sturge Weber syndrome, hemimegalencephaly, or hemispheric stroke with associated hemiparesis.

However, the surgical option is not considered if cortical resection can create a new unacceptable neurological deficit, in cases of diffuse brain damage or the presence of any degenerative or metabolic disease [5].

\section{Prognosis}

Despite advances in early diagnosis and appropriate treatment, SW prognosis remains poor; $75-90 \%$ of patients have mental retardation and $50-60 \%$ of children have recurrent seizures at five years of age. Approximately $27-50 \%$ develop Lennox-Gastaut syndrome. The mortality rate varies between 3-33\% [5].

\section{Clinical Summary}

\section{Hospital Outcomes}

It is 4 years female patient who was born via vaginal without eventualities. No maternal complications during pregnancy, prolonged delivery, Apgar 8/9. 
During the first 4 hours life, baby presented irritable crying and poor suction reflex and was admitted to the Neonatology where she had seizures in the first 24 hours of life, treated with anticonvulsivants. During hospitalization in neonatology (15 days) baby did not have seizures again and she kept hypoactive due medication, and was discharged with Phenobarbital.

Examns in Neonatology:

1. Lumbar puncture: normal

2. Ultrasound transfontanelar: normal

No more seizures during first 3 months but showed a delay development and growth for the age. Due this, a Neurology evaluation decided increase dose of Phenobarbital adding to treatment Psicosoma and Diazepam (these last two to keep calm the baby because most time she was irritable ) giving a diagnosis of:

\section{Microcephaly}

2. Left Predominance Cerebral Paralysis and

3. Static lesion of the central nervous system.

4. Gastroenterology disease, asking an evaluation for Gastroenterology.

The Gastroenterology assessment diagnosticated:

1. Gastroesophageal Reflux.

2. Allergy to milk protein

The patient kept well neurologically and was feeding with hydrolyzed formula, the target was the gaining weight and improve her nutrition state.

At one and a half years of age, parents notice certain involuntary movements in the hands and feet, accompanied by small spasms that immediately made the baby cry (parents believe that the baby is nervous and scared). When these episodes continue, the baby is valued by Pediatrician, who recommends to a new assessment by a Neurologist Pediatrician, who concludes with an EEG that are convulsions of difficult control, the management was only to increase doses of anticonvulsants.

A new assessment for other Neurologist Pediatrician, realized an EEG with the following findings: During the sleep phase, it is observed base slow ritm, spindles of sleep sporadic and asynchronous. At the same time, there is frequent discharge of acute wave-tip in both hemispheres, with secondary generalization, with suppression pattern (Hypsarrhythmia pattern).

The complete assessment diagnosed the patient with a West Syndrome due the triad of:

- Infantile spasms that appear in salvos.
- Alteration of psychomotor development.

- E.E.G. Intercritical: Hypsarrhythmia.

New treatment is established:

- Valproic acid (high doses)

- Diazepam

- Lamotrigine

- Neurostat

Then added Vigabatrin.

With improvement of seizures before November 2016 when patient had a relapse, several seizures per day, Considering in that moment an interface between West syndrome and Lennox Gastaut but EEG showed Hypsarrythmiatipical of West Syndrome and started therapy with immunoglobulins.

The patient needed Nissen Fundoplication and Gastrostomy to be feeded because she was losing weight and had a bad nutritional state.

\section{Medical Exams}

Transfontanellar ultrasound at birth: Normal

Transfontanellar ultrasound July/04/2013: Normal

MRI September/02/2013: Increased size of subarachnoid spaces Slight supratentorial ventricular dilatation

Esophagogastroduodenal series April/28/2014: Dilated stomach with slow gastric emptying. There was no gastroesophageal reflux EEG March/12/2015: Hypsiarritmic pattern

\section{Conclusion}

In this case we don't have enough data to speculate on etiology. The fact that seizures occurred within first 24 hours of life raise suspicion for perinatal event (bleed, hypoxia). Metabolic disease is often hallmarked by progressive symptoms, failure to thrive, microcephaly, etc. Due the disorder is static a metabolic disease is still possible but less likely.

\section{References}

[1]. Watanabe K. Recent advances and some problems in the delineation of epileptic syndromes in children. Brain Dev. 1996 Nov 1;18(6):423-37.

[2]. Pavone P, Striano P, Falsaperla R, Pavone L, Ruggieri M. Infantile spasms syndrome, Westsyndrome and related phenotypes: What weknow in 2013. Brain Dev. 2014 Oct 31;36(9):739-751.

[3]. West WJ. On a peculiar form of infantile convulsions. The Lancet. $1841 \mathrm{Feb}$ 13;35(911):724-5.

[4]. Wheless J, Gibson P, Rosbeck K, Hardin M, O'Dell C, Whittemore V, et al. Infantile spasms (West syndrome):update and resources for pediatricians and providers to share with parents. BMC Pediatrics. 2012 Jul 25;12(1):108.

[5]. García RA, Jimenez A, Mathur S, Ballot J, Eff-Darwich A, Jimenez-Reyes SJ, et al. Update on g-mode research. Astronomical Notes. 2008 May 14;329(5):476-484. 